\title{
Nuclear Magnetic Relaxation of Poly(n-hexyl isocyanate) in Dilute Solution. Effects of Chain Stiffness
}

\author{
Masayuki NAKATsujI, Takenao YoshizAKI, ${ }^{\dagger}$ and Hiromi YAmaKawA \\ Department of Polymer Chemistry, Kyoto University, Katsura, Nishikyo-ku, Kyoto 615-8510, Japan
}

(Received April 8, 2005; Accepted April 26, 2005; Published July 15, 2005)

\begin{abstract}
The spin-lattice relaxation time $T_{1}$, spin-spin relaxation time $T_{2}$, and nuclear Overhauser enhancement NOE were determined from nuclear magnetic relaxation measurements on five well-fractionated samples of poly( $n$-hexyl isocyanate) in the range of weight-average molecular weight $M_{\mathrm{w}}$ from $1.65 \times 10^{4}$ to $5.71 \times 10^{4}$ in $n$-hexane at $25^{\circ} \mathrm{C}$. It is found that $T_{1}$ increases and $T_{2}$ decreases monotonically with increasing $M_{\mathrm{w}}$ in the range of $M_{\mathrm{w}}$ examined, while NOE is almost independent of $M_{\mathrm{w}}$. Such behavior may be regarded as arising from effects of chain stiffness. A comparison is then made of the present data for $T_{1}, T_{2}$, and NOE with the theory developed for the generalized KratkyPorod (KP) wormlike chain as a special case of the helical wormlike chain, and it is shown that the theory may almost quantitatively explain the data, using the established values of the KP model parameters. A comparison is also made of the present data with the theoretical values calculated from the equations of Woessner for the equivalent, rigid prolate spheroid. [DOI 10.1295/polymj.37.535]

KEY WORDS Poly(n-hexyl isocyanate) / Nuclear Magnetic Relaxation / Spin-Lattice Relaxation Time / Spin-Spin Relaxation Time / Nuclear Overhauser Enhancement / Generalized KratkyPorod Wormlike Chain / Helical Wormlike Chain /
\end{abstract}

As is well known, the spin-lattice relaxation time $T_{1}$, spin-spin relaxation time $T_{2}$, and nuclear Overhauser enhancement NOE for ${ }^{13} \mathrm{C}$ nuclei may be expressed in terms of time-correlation functions of components of the dyadic of the internuclear vector ${ }^{13} \mathrm{C} \rightarrow{ }^{1} \mathrm{H}$ from a ${ }^{13} \mathrm{C}$ nucleus to a bonded ${ }^{1} \mathrm{H}$ one if we simply assume that the nuclear magnetism of ${ }^{13} \mathrm{C}$ relaxes only due to the heteronuclear dipolar interaction. ${ }^{1,2} \mathrm{We}$ have made so far experimental studies of $T_{1}$ and NOE for backbone ${ }^{13} \mathrm{C}$ of typical flexible polymers such as atactic polystyrene, ${ }^{3}$ atactic poly(methyl methacrylate), ${ }^{4}$ isotactic poly(methyl methacrylate),${ }^{5}$ and atactic poly $(\alpha$-methylstyrene $) .{ }^{6}$ In the case of flexible polymers, tumbling motions of repeat units are hardly coupled with the entire chain motion, so that values of $T_{1}, T_{2}$, and NOE are almost independent of the molecular weight $M$ except for oligomers with very small $M$. In the case of semiflexible polymers, on the other hand, the former motions may be considered to be coupled with the latter even in the range of rather large $M$, and therefore the dependence of $T_{1}, T_{2}$, and NOE on $M$ must be different from that for flexible polymers. Unfortunately, however, such literature data for typical semiflexible polymers are not available except those obtained by Budd et al. ${ }^{7}$ for $T_{1}$ and $T_{2}$ for $\operatorname{poly}(\gamma$-benzyl L-glutamate) (PBLG). In the present paper, therefore, we examine experimentally the dependence on $M$ of $T_{1}, T_{2}$, and also NOE for poly(n-hexyl isocyanate) (PHIC) as another example of semiflexible polymers. We note that
DuPré and $\mathrm{Wang}^{8}$ have determined $T_{1}, T_{2}$, and NOE for a PHIC sample with the weight-average molecular weight $M_{\mathrm{w}}=3.39 \times 10^{4}$, and therefore have not examined the dependence of these quantities on $M_{\mathrm{w}}$.

PHIC is a typical semiflexible polymer whose molecular characterization has almost completely been done by an analysis of dilute solution properties on the basis of the Kratky-Porod (KP) wormlike chain model. ${ }^{9,10}$ Specifically, the KP model parameters for PHIC, i.e., the static stiffness parameters $\lambda^{-1}$, the shift factor $M_{\mathrm{L}}$ as defined as the molecular weight per unit contour length of the KP chain, and the hydrodynamic diameter $d$, have been determined rather unambiguously. ${ }^{11}$ Thus, from a comparison of present experimental data with the theory previously ${ }^{12}$ developed for the generalized KP chain [a special case of the helical wormlike (HW) chain] ${ }^{10}$ by the use of the established values of the KP model parameters, ${ }^{11}$ we may verify its validity. This is the main purpose of the present paper.

A remark should be made here on the ${ }^{13} \mathrm{C}$ atoms of PHIC under observation. In their study of PBLG, Budd et ll $^{7}$ have observed signals from backbone ${ }^{13} \mathrm{C}^{\alpha}$ atoms in order to trace main-chain motions. In the present case of PHIC, however, the backbone carbon atoms are only carbonyl ones and their nuclear magnetism never relaxes due to the heteronuclear dipolar interaction, so that main-chain motions cannot then be directly traced. Thus, following DuPré and Wang, ${ }^{8}$ we assume that the bond vector from the

${ }^{\dagger}$ To whom correspondence should be addressed (E-mail: yoshizaki@molsci.polym.kyoto-u.ac.jp). 
Table I. Values of $M_{\mathrm{w}}, x_{\mathrm{w}}$, and $M_{\mathrm{w}} / M_{\mathrm{n}}$ for $\operatorname{poly}(n$-hexyl isocyanate)

\begin{tabular}{lccc}
\hline Sample & $M_{\mathrm{w}}$ & $x_{\mathrm{w}}$ & $M_{\mathrm{w}} / M_{\mathrm{n}}$ \\
\hline PHIC1 & $1.65 \times 10^{4}$ & 130 & 1.10 \\
PHIC2b & $2.66 \times 10^{4}$ & 209 & 1.07 \\
PHIC3 & $2.90 \times 10^{4}$ & 228 & 1.10 \\
PHIC4 & $4.71 \times 10^{4}$ & 371 & 1.04 \\
PHIC5 & $5.71 \times 10^{4}$ & 450 & 1.05 \\
\hline
\end{tabular}

$n$-hexyl (side-chain) $\mathrm{C}$ atom next to a (main-chain) nitrogen atom, which we designate by $\mathrm{C} 1$, to a bonded $\mathrm{H}$ atom is rigidly attached to the backbone, and make a comparison of experimental results for ${ }^{13} \mathrm{C} 1$ with the theory.

\section{EXPERIMENTAL}

\section{Materials}

All the five PHIC samples PHIC1, PHIC2b, PHIC3, PHIC4, and PHIC5 used in this work are the same as those used in a previous study of the mean-square optical anisotropy $\left\langle\Gamma^{2}\right\rangle{ }^{13}$ The original sample from which the five test samples were separated by fractional precipitation had been prepared following the procedure of Aharoni, ${ }^{14}$ i.e., polymerized in a toluene/ $\mathrm{N}, \mathrm{N}$-dimethylformamide $(1: 1)$ mixture at $-78^{\circ} \mathrm{C}$ with sodium cyanide as an initiator and methanol as a terminator. In Table I have been reproduced the values of $M_{\mathrm{w}}$, the weight-average degree of polymerization $x_{\mathrm{w}}$, and the ratio $M_{\mathrm{w}} / M_{\mathrm{n}}$ of $M_{\mathrm{w}}$ to the numberaverage molecular weight $M_{\mathrm{n}}$ for the five samples from Table I of ref 13.

The solvent $n$-hexane used for nuclear magnetic relaxation measurements was purified according to a standard procedure prior to use.

\section{Nuclear Magnetic Relaxation}

$T_{1}$ and $T_{2}$ were determined for ${ }^{13} \mathrm{C} 1$ of all the five samples by the inversion-recovery method with a pulse sequence $\pi-\tau-\pi / 2$ on a JEOL JNM GX-400 spectrometer at $100.5 \mathrm{MHz}$. NOE for ${ }^{13} \mathrm{C} 1$ for each sample was evaluated from the ratio of the integrated intensity of its peak obtained with complete noise decoupling of protons to that obtained with gated decoupling only during data acquisition. A pulse delay was taken to be longer than $1.5 \mathrm{~s}$, so that it is $c a .8$ times as long as $T_{1}$ for ${ }^{13} \mathrm{C} 1$ under observation. Measurements were carried out on solutions in $n$-hexane at $25^{\circ} \mathrm{C}$ with a lock signal obtained from an external $\mathrm{C}_{6} \mathrm{D}_{12}$ tube. The values of the polymer mass concentration $c$ of the test solutions are $0.009_{7}, 0.020_{1}$, $0.046_{4}, 0.058_{4}$, and $0.051_{0} \mathrm{~g} / \mathrm{cm}^{3}$ for PHIC1, PHIC2b, PHIC3, PHIC4, and PHIC5, respectively, which were calculated from the weight fractions with the densities
Table II. Values of $T_{1}, T_{2}$, and NOE for $\operatorname{poly}(n$-hexyl isocyanate) in $n$-hexane at $25^{\circ} \mathrm{C}$

\begin{tabular}{lccc}
\hline Sample & $T_{1}, \mathrm{~s}$ & $T_{2}, \mathrm{~s}$ & $\mathrm{NOE}$ \\
\hline PHIC1 & $0.16_{7}$ & $0.012_{9}$ & $1.0_{8}$ \\
PHIC2b & $0.17_{0}$ & $0.008_{1}$ & $1.1_{3}$ \\
PHIC3 & $0.18_{6}$ & $0.005_{0}$ & $1.1_{2}$ \\
PHIC4 & $0.19_{9}$ & $0.003_{9}$ & $1.3_{5}$ \\
PHIC5 & $0.21_{8}$ & $0.003_{4}$ & $1.2_{0}$ \\
\hline
\end{tabular}

of the solutions. The solutions were not degassed since $T_{1}$ 's of interest never exceeded $2 \mathrm{~s}$, as shown in the next (RESULTS) section. We note that the resonance peak of ${ }^{13} \mathrm{C} 1(48.8 \mathrm{ppm})$ is well separated from those of ${ }^{13} \mathrm{C}$ in the solvent $n$-hexane and that $T_{1}$ and $T_{2}$ have been estimated by single-exponential fitting.

\section{RESULTS}

In Table II are given the values of $T_{1}, T_{2}$, and NOE for ${ }^{13} \mathrm{C} 1$ for the five PHIC samples PHIC1 (at $c=0.009_{7} \mathrm{~g} / \mathrm{cm}^{3}$ ), PHIC2b (at $c=0.020_{1} \mathrm{~g} / \mathrm{cm}^{3}$ ), PHIC3 (at $c=0.046_{4} \mathrm{~g} / \mathrm{cm}^{3}$ ), PHIC4 (at $c=0.058_{4}$ $\mathrm{g} / \mathrm{cm}^{3}$ ), and PHIC5 (at $c=0.051_{0} \mathrm{~g} / \mathrm{cm}^{3}$ ) in $n$ hexane at $25^{\circ} \mathrm{C}$ and at the Larmor frequency 100.5 $\mathrm{MHz}$ of ${ }^{13} \mathrm{C}$. Figure 1 shows plots of $T_{1}, T_{2}$, and NOE for ${ }^{13} \mathrm{C} 1$ so obtained against $M_{\mathrm{w}}$. The circles,

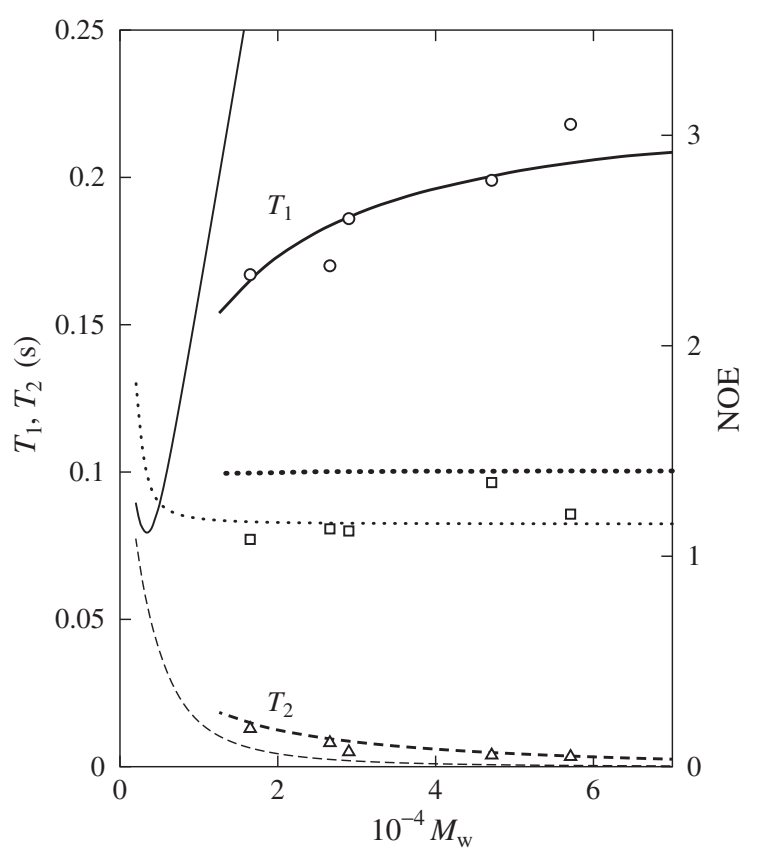

Figure 1. Plots of $T_{1}, T_{2}$, and NOE against $M_{\mathrm{w}}$ for the ${ }^{13} \mathrm{C} 1$ atoms for PHIC in $n$-hexane at $25^{\circ} \mathrm{C}$ : (○) $T_{1} ;(\triangle) T_{2}$; $(\square)$ NOE. The heavy solid, dashed, and dotted curves represent the generalized KP theoretical values of $T_{1}, T_{2}$, and NOE, respectively, and the light curves represent the respective theoretical values for the rigid prolate spheroid (see the text). 
triangles, and squares represent the experimental values of $T_{1}, T_{2}$, and NOE, respectively. In the figure, the heavy solid, dashed, and dotted curves represent the generalized KP theoretical values of $T_{1}, T_{2}$, and NOE, respectively, and the light curves represent the respective theoretical values for the rigid prolate spheroid, which are obtained and discussed in the next discussion section.

It is clearly seen that $T_{1}$ increases and $T_{2}$ decreases monotonically with increasing $M_{\mathrm{w}}$ in the range of $M_{\mathrm{w}}$ examined as in the case of PBLG. ${ }^{7}$ Such behavior of $T_{1}$ and $T_{2}$ may be regarded as arising from effects of chain stiffness. As for NOE, it seems almost independent of $M_{\mathrm{w}}$ in the range of $M_{\mathrm{w}}$ examined.

DuPré and Wang ${ }^{8}$ have determined $T_{1}, T_{2}$, and NOE for ${ }^{13} \mathrm{C} 1$ for the PHIC sample with $M_{\mathrm{w}}=3.39 \times 10^{4}$ in chloroform- $d$ and in toluene at $30^{\circ} \mathrm{C}$ and at the Larmor frequencies 75 and $126 \mathrm{MHz}$ of ${ }^{13} \mathrm{C}$. We note that the concentrations of their test solutions are $c a$. $0.06 \mathrm{~g} / \mathrm{cm}^{3}$ and are nearly of the same order as ours. The values of $T_{1}, T_{2}$, and NOE obtained in chloroform- $d$ are $0.145 \mathrm{~s}, 0.005 \mathrm{~s}$, and 1.59 , respectively, at $75 \mathrm{MHz}$ and $0.290 \mathrm{~s}, 0.005 \mathrm{~s}$, and 1.13, respectively, at $126 \mathrm{MHz}$, and those in toluene are $0.100 \mathrm{~s}, 0.005 \mathrm{~s}$, and 1.63 , respectively, at $75 \mathrm{MHz}$. As for the results in toluene at $126 \mathrm{MHz}$, they have reported only the values $0.342 \mathrm{~s}$ and $0.003 \mathrm{~s}$ of $T_{1}$ and $T_{2}$, respectively. Since their solvent conditions and Larmor frequencies are different from those adopted in the present measurements, we cannot directly compare the present results with the above literature data. We only note that the present and literature data are not very inconsistent with each other, being of the same order of magnitude.

\section{DISCUSSION}

\section{HW Theory}

Before proceeding to make a comparison of the present data for $T_{1}, T_{2}$, and NOE with the generalized KP chain theory, ${ }^{10,12}$ we briefly summarize its relevant results.

The generalized KP chain is a special case of the HW chain which is defined as an elastic wire. ${ }^{10}$ The equilibrium conformational behavior of the HW chain may in general be described by four parameters: the constant differential-geometrical curvature $\kappa_{0}$ and torsion $\tau_{0}$ of the characteristic helix taken at minimum zero of its elastic energy and the bending and torsional force constants. In the particular case of $\kappa_{0}=0$, the characteristic helix becomes a straight line, and then the HW chain with $\kappa_{0}=0$ is referred to as the generalized KP chain. Further, for convenience, the generalized KP chain is classified into two types: one with $\tau_{0} \neq 0$ (KP1) and the other with $\tau_{0}=0$ (KP2). In an application of the HW chain to flexible polymers, the bending and torsional force constants are set equal to each other (Poisson's ratio $\sigma=0$ ), for simplicity, and these two parameters may then be reduced to the single (static stiffness) parameter $\lambda^{-1}$. Although the two force constants should not necessarily be set equal to each other in the case of the generalized KP chain, we do so as in the previous analysis ${ }^{12}$ of the PBLG data. ${ }^{7}$ Then the model parameters describing the generalized KP chain in the present case are $\lambda^{-1}$ and $\tau_{0}$. The basic equations necessary for the present analysis are in principle the same as those for the HW chain $(\sigma=0)$ used in the previous analy$\operatorname{ses}^{3-6}$ of the data for the typical flexible polymers.

In order to apply the HW (or generalized KP) chain to an analysis of dynamic properties, we use its dynamic version, the discrete HW (or generalized KP) chain, ${ }^{10}$ which is composed of $N$ identical rigid subbodies, each with the translational and rotatory friction coefficients $\zeta_{\mathrm{t}}$ and $\zeta_{\mathrm{r}}$. The subbodies are successively joined with bonds of fixed length $a$, and the potential energy as a function of the orientation of a subbody relative to the preceding one is properly chosen so that the equilibrium conformational behavior of the dynamic version may reproduce well that of the original one.

As mentioned in the introduction, we assume that the nuclear magnetic spin (of ${ }^{13} \mathrm{C} 1$ atom in the present case) relaxes only due to the heteronuclear dipolar interaction between two unlike spins $I$ and $S$ with spin $I$ observed and spin $S$ irradiated (i.e., spins of ${ }^{13} \mathrm{C}$ and ${ }^{1} \mathrm{H}$ atoms, respectively, in the present case, so that $I=S=1 / 2$ ) and with the internuclear distance $r$ between them. Then $T_{1}, T_{2}$, and NOE for the single (discrete) generalized KP chain [i.e., the single (discrete) HW chain with $\kappa_{0}=0$ ] may be given by ${ }^{10,12}$

$$
\begin{aligned}
T_{1}^{-1}= & (1 / 20) K^{2} r^{-6}\left[J_{0}\left(\omega_{S}-\omega_{I}\right)+3 J_{1}\left(\omega_{I}\right)\right. \\
& \left.+6 J_{2}\left(\omega_{S}+\omega_{I}\right)\right] \\
T_{2}^{-1}= & (1 / 40) K^{2} r^{-6}\left[4 J_{0}(0)+J_{0}\left(\omega_{S}-\omega_{I}\right)\right. \\
& \left.+3 J_{1}\left(\omega_{I}\right)+6 J_{1}\left(\omega_{S}\right)+6 J_{2}\left(\omega_{S}+\omega_{I}\right)\right] \\
\mathrm{NOE}=1 & +\frac{\gamma_{S}}{\gamma_{I}} \\
\times & {\left[\frac{6 J_{2}\left(\omega_{S}+\omega_{I}\right)-J_{0}\left(\omega_{S}-\omega_{I}\right)}{J_{0}\left(\omega_{S}-\omega_{I}\right)+3 J_{1}\left(\omega_{I}\right)+6 J_{2}\left(\omega_{S}+\omega_{I}\right)}\right] }
\end{aligned}
$$

with

$$
K=\hbar \gamma_{I} \gamma_{S}
$$

where $\gamma_{I}$ and $\gamma_{S}$ are the gyromagnetic ratios of spins $I$ and $S$, respectively, $\omega_{I}$ and $\omega_{S}$ are the respective Larmor angular frequencies, $\hbar$ is Dirac's constant (Planck's constant divided by $2 \pi)$, and $J_{m}(\omega)(m=$ $0,1,2)$ is the spectral density written in the form ${ }^{10,12}$ 


$$
J_{m}(\omega)=2 \sum_{k=1}^{N}\left(Q_{p k}^{0}\right)^{2} \sum_{j=-2}^{2} \frac{A_{2, k}^{j} \tau_{2, k}^{j}}{1+\left(\omega \tau_{2, k}^{j}\right)^{2}}
$$

with $p$ indicating the subbody number and with

$$
\begin{gathered}
Q_{p k}^{0}=[2 /(N+1)]^{1 / 2} \sin [\pi p k /(N+1)] \\
\tau_{2, k}^{j}=1 / \lambda_{2, k}^{j}
\end{gathered}
$$

We note that $J_{m}$ is independent of $m$. The quantities $\lambda_{2, k}^{j}$ in eq 7 are the eigenvalues of the matrix representation of the diffusion operator associated with the subspace 2(1) (of full Hilbert space) spanned by the basis set with the "total angular momentum quantum number" $L=2$ and the number of "excited" subbodies $n=1$, and the coefficients $A_{2, k}^{j}$ in eq 5 are given by eq 29 of ref 12 . We use the eigenvalues $\lambda_{2, k}^{j}$ in the crude subspace approximation given by eq B5 of ref 15 as in the previous study ${ }^{12}$ of nuclear magnetic relaxation of semiflexible polymers. Thus $\lambda_{2, k}^{j}$ may readily be calculated for given values of $N$ and the five (discrete) generalized KP model parameters: $\lambda^{-1}$, $\tau_{0}, a, \zeta_{\mathrm{t}}$, and $\zeta_{\mathrm{r}}$. We note that the coefficient $A_{2, k}^{j}$ depends on the parameters $\lambda^{-1}$ and $\tau_{0}$ and also on the absolute value of the angle $\alpha$ between the $\mathrm{C}-\mathrm{H}$ internuclear vector and the KP chain contour.

When two or more spins $S$ contribute to the relaxation of spin $I$ under consideration, the above formula 1-3 should be modified. If the internuclear distances of all spin pairs are the same, then $T_{1}, T_{2}$, and NOE are given by eqs $1-3$, respectively, but with the sum of spectral densities $J_{m}^{(i)}$ over spin pair $i$ in place of $J_{m}$.

\section{Dependence of $T_{1}, T_{2}$, and NOE on $M_{w}$}

As in the previous study of $\left\langle\Gamma^{2}\right\rangle,{ }^{13}$ we assume that the two kinds of rotation angles around the $\mathrm{C}-\mathrm{N}$ bonds in the main chain of PHIC take the values given by Troxell and Scheraga ${ }^{16}$ for poly(methyl isocyanate), and also that each $n$-hexyl group takes the all-trans conformation and is attached to the $\mathrm{N}$ atom with its axis of symmetry making a right angle with the helix axis (with the minimum energy), for simplicity. Then the backbone of PHIC takes the Schmueli-TraubRosenheck ${ }^{17} 8_{3}$ helix, ${ }^{16,18-20}$ and the equilibrium conformational behavior of its helix axis (without consideration of the torsion) may be well described by the (original $^{9}$ ) KP chain with $\lambda^{-1}=840 \AA$ and $M_{\mathrm{L}}=$ $71.5 \AA^{-1} .^{18}$ If we take the repeat unit of PHIC as the subbody composing the discrete model, the bond length $a$ may then be calculated to be $1.78 \AA$ from the relation $a \simeq M_{0} / M_{\mathrm{L}}$ with the above value of $M_{\mathrm{L}}$ and the value 127 of the molecular weight $M_{0}$ of the repeat unit. Further, the localized Cartesian coordinate system $(\xi, \eta, \zeta)^{10}$ affixed to each subbody with its $\zeta$ axis in the direction of the chain contour (the $8_{3}$ helix axis in the present case) rotates around the helix axis with the pitch $h=4.74 \AA$ (of the present $8_{3}$ helix), so that the KP1 torsion $\tau_{0}(=2 \pi / h)$ becomes equal to $1.33 \AA^{-1}$ (see also Figure $4.4 \mathrm{~b}$ of ref 10 ). The dimensionless parameters $r_{1} \equiv \zeta_{\mathrm{t}} / 3 \pi \eta_{0} a$ and $r_{2} \equiv \zeta_{\mathrm{r}} / a^{2} \zeta_{\mathrm{t}}$ with $\eta_{0}$ the solvent viscosity are used in place of $\zeta_{\mathrm{t}}$ and $\zeta_{\mathrm{r}}$ themselves. The values of $r_{1}$ is set equal to 1 , as usually done, ${ }^{3-6,12,21}$ and $r_{2}$ as an adjustable parameter is determined from a best fit of the theoretical values to the data for $T_{1}$, as previously done. ${ }^{12}$

Each ${ }^{13} \mathrm{C} 1$ atom of PHIC has two bonded ${ }^{1} \mathrm{H}$ atoms and the two angles $\alpha$ 's, each between one of the two internuclear vectors ${ }^{13} \mathrm{C} 1 \rightarrow{ }^{1} \mathrm{H}$ and the chain contour (the $8_{3}$ helix axis), are estimated to be $39^{\circ}$ and $71^{\circ}$ for the chain conformation mentioned above. Then the observed $T_{1}$ and $T_{2}$ must be compared with one half of the harmonic mean of the two theoretical values calculated from eqs 1 and 2 with eqs 4-7 with $\alpha=$ $39^{\circ}$ and $71^{\circ}$. As for NOE, we simply compare the observed value with the arithmetic mean of the two theoretical values calculated from eqs 3-7 with $\alpha=$ $39^{\circ}$ and $71^{\circ}$, which gives a good approximate value, as done by DuPré and Wang. ${ }^{8}$

In Figure 1, the heavy solid, dashed, and dotted curves represent the generalized KP theoretical values of $T_{1}, T_{2}$, and NOE, respectively, so calculated from eqs 1-7 with $r_{2}=320$ along with the above mentioned values of the parameters, where $N$ has been converted to $M_{\mathrm{w}}$ by $M_{\mathrm{w}}=N M_{0},\left(Q_{p k}^{0}\right)^{2}$ in eq 5 has been replaced by $N^{-1}$, and the value $0.299 \mathrm{cP}$ has been used for $\eta_{0}$ for $n$-hexane at $25^{\circ} \mathrm{C}$. The crude subspace approximation is invalid for small $M$ (for $N \lesssim 100$ ), so that we have shown the theoretical values only for $N \geq 99$. It is seen that the theory gives almost a quantitative explanation of the experimental results within experimental error. We then find the hydrodynamic diameter $d$ of the KP cylinder having the same hydrodynamic volume as the discrete generalized KP chain to be equal to $19 \AA$. It has been calculated from eqs 35-38 of ref 21 with the above values of $r_{2}$ and $a$, assuming, as in the previous study, ${ }^{12}$ that the subbody of the latter is an oblate spheroid having the rotation axis of length $a$ and the diameter $d_{\mathrm{s}}$. (We note that $d_{\mathrm{s}}=23 \AA$.) This value of $d$ is fairly consistent with the values 16 and $25 \AA$ determined from the intrinsic viscosity and sedimentation coefficient, ${ }^{18}$ respectively.

It is pertinent to make here a remark on the assumption of $\sigma=0$. In the case of duplex DNA, especially in the problem in which its torsion must be explicitly considered, ${ }^{10,22} \sigma$ is usually chosen to be a negative value $(\sim-0.3)$, the torsional constant being larger than the bending one. For single-helix chains such as PHIC and PBLG, the torsional constant may not be considered to be larger than the bending one, and therefore the assumption of $\sigma=0$ seems rather reasonable. 
For comparison, the light solid, dashed, and dotted curves in Figure 1 represent the theoretical values of $T_{1}, T_{2}$, and NOE, respectively, calculated from the equations of Woessner ${ }^{23}$ for the equivalent, rigid prolate spheroid having the rotation axis of length $d_{\|}$ ( $\left.=M_{\mathrm{w}} / M_{\mathrm{L}}\right)$ and the diameter $d_{\perp}$. We have set $d_{\perp}=(3 / 2)^{1 / 2} d$ so that the hydrodynamic volume of the spheroid is identical to that of the KP cylinder with $d=19 \AA$ A. His equations read eqs $1-3$ with the spectral density given by

$$
\begin{aligned}
J_{m}(\omega)= & \frac{\left(3 \cos ^{2} \alpha-1\right)^{2}}{2} \frac{\tau_{1}}{1+\left(\omega \tau_{1}\right)^{2}} \\
& +\frac{3 \sin ^{2}(2 \alpha)}{2} \frac{\tau_{2}}{1+\left(\omega \tau_{2}\right)^{2}} \\
& +\frac{3 \sin ^{4} \alpha}{2} \frac{\tau_{3}}{1+\left(\omega \tau_{3}\right)^{2}}
\end{aligned}
$$

where $\alpha\left(=39^{\circ}\right.$ or $\left.71^{\circ}\right)$ is the angle between the internuclear vector and the rotation axis, and $\tau_{i}(i=1,2$, 3 ) is the relaxation time defined by

$$
\begin{aligned}
\tau_{1} & =\left(6 D_{\perp}\right)^{-1} \\
\tau_{2} & =\left(5 D_{\perp}+D_{\|}\right)^{-1} \\
\tau_{3} & =\left(2 D_{\perp}+4 D_{\|}\right)^{-1}
\end{aligned}
$$

with $D_{\|}$and $D_{\perp}$ the rotatory diffusion coeffcients of the spheroid around the rotation and transverse axes, respectively, given by ${ }^{24,25}$

$$
\begin{aligned}
\frac{\pi \eta_{0} d_{\perp}{ }^{3} D_{\|}}{k_{\mathrm{B}} T} & =\frac{3}{2\left(\epsilon^{2}-1\right)}[\epsilon-F(\epsilon)] \\
\frac{\pi \eta_{0} d_{\perp}{ }^{3} D_{\perp}}{k_{\mathrm{B}} T} & =\frac{3}{2\left(\epsilon^{4}-1\right)}\left[\left(2 \epsilon^{2}-1\right) F(\epsilon)-\epsilon\right]
\end{aligned}
$$

In eqs 10 and $11, k_{\mathrm{B}}$ is the Boltzmann constant, $T$ is the absolute temperature, and $F(\epsilon)$ is the function of the ratio $\epsilon=d_{\|} / d_{\perp}$ given by

$$
F(\epsilon)=\left(\epsilon^{2}-1\right)^{-1 / 2} \cosh ^{-1} \epsilon
$$

for $\epsilon>1$ (prolate spheroid). It is seen that the behavior of the present data for PHIC cannot be explained by the completely rigid spheroid as in the previous analysis of the PBLG data. ${ }^{12}$

Finally, we note that the values $39^{\circ}$ and $71^{\circ}$ adopted here for $\alpha$ in the calculation of the theoretical values for the generalized KP chain and the rigid spheroid are different from the values $81.3^{\circ}$ and $118.6^{\circ}$ used by DuPré and Wang, ${ }^{8}$ but that the theoretical values are not appreciably altered even if the latter values of $\alpha$ are used in place of the former.

\section{CONCLUSIONS}

The spin-lattice relaxation time $T_{1}$, spin-spin relaxation time $T_{2}$, and nuclear Overhauser enhancement
NOE were determined for PHIC in $n$-hexane at $25^{\circ} \mathrm{C}$, probing the $n$-hexyl ${ }^{13} \mathrm{C}$ atoms bonded to the main-chain nitrogen atoms. It has been found that $T_{1}$ increases and $T_{2}$ decreases monotonically with increasing $M_{\mathrm{w}}$ in the range of $M_{\mathrm{w}}$ examined. Such behavior may be regarded as arising from effects of chain stiffness. It has then been shown that the generalized KP theory (a special case of the HW one) may almost quantitatively explain such behavior of the experimental data within experimental error.

Acknowledgment. This research was supported in part by the 21st century COE program "COE for a United Approach to New Materials Science" from the Ministry of Education, Culture, Sports, Science, and Technology, Japan.

\section{REFERENCES}

1. I. Solomon, Phys. Rev., 99, 599 (1955).

2. A. Abragam, "The Principles of Nuclear Magnetism," Oxford University Press, London, U.K., 1961.

3. Y. Takaeda, T. Yoshizaki, and H. Yamakawa, Macromolecules, 27, 4248 (1994).

4. Y. Takaeda, T. Yoshizaki, and H. Yamakawa, Macromolecules, 28, 682 (1995).

5. Y. Naito, N. Sawatari, Y. Takaeda, T. Yoshizaki, and H. Yamakawa, Macromolecules, 30, 2751 (1997).

6. M. Osa, H. Ueda, T. Yoshizaki, and H. Yamakawa, Polym. J., 37, 14 (2005).

7. P. M. Budd, F. Heatley, T. J. Holton, and C. Price, J. Chem. Soc., Faraday Trans. 1, 77, 759 (1981).

8. D. B. DuPré and H. Wang, Macromolecules, 25, 7155 (1992).

9. O. Kratky and G. Porod, Recl. Trav. Chim. Pays-Bas, 68, 1106 (1949).

10. H. Yamakawa, "Helical Wormlike Chains in Polymer Solutions," Springer, Berlin, 1997.

11. T. Norisuye, Prog. Polym. Sci., 18, 543 (1993).

12. H. Yamakawa and M. Fujii, J. Chem. Phys., 81, 997 (1984).

13. M. Nakatsuji, Y. Ogata, M. Osa, T. Yoshizaki, and H. Yamakawa, Macromolecules, 34, 8512 (2001).

14. S. M. Aharoni, Macromolecules, 13, 409 (1979).

15. H. Yamakawa, T. Yoshizaki, and J. Shimada, J. Chem. Phys., 78, 560 (1983).

16. T. C. Troxell and H. A. Scheraga, Macromolecules, 4, 528 (1971).

17. U. Schmueli, W. Traub, and K. Rosenheck, J. Polym. Sci., A-2, 7, 515 (1969).

18. H. Murakami, T. Norisuye, and H. Fujita, Macromolecules, 13, 345 (1980).

19. S. Lifson, C. E. Felder, and M. M. Green, Macromolecules, 25, 4142 (1992).

20. M. M. Green, N. C. Peterson, T. Sato, A. Teramoto, R. Cook, and S. Lifson, Science, 268, 1860 (1995).

21. T. Yoshizaki and H. Yamakawa, J. Chem. Phys., 81, 982 (1984).

22. a) J. Shimada and H. Yamakawa, Macromolecules, 17, 689 
M. NAKAtsuji, T. YoshizaKi, and H. YAmaKawa

(1984).

b) J. Shimada and H. Yamakawa, J. Mol. Biol., 184, 319 (1985).
23. D. E. Woessner, J. Chem. Phys., 37, 647 (1962).

24. G. B. Jeffery, Proc. R. Soc. London, Ser. A, 102, 161 (1922).

25. F. Perrin, J. Phys. Radium, 7, 1 (1936). 